films in which the faults are planar and are parallel to the incident electron beam. Kinematically the intensity expression takes the form

$$
I_{\text {diff. }}=\left|F_{\text {kin }}(\mathbf{u})\right|^{2} \cdot D(\mathbf{u}),
$$

where $D(\mathbf{u})$ is the Fourier transform of the correlation function describing the disorder of the structural units and $F_{\mathrm{kin}}(\mathbf{u})$ is the kinematical structure amplitude for the structural unit. Then, within the limits of validity of the column approximation the dynamical intensity is given by

$$
I_{\text {diff. }}^{\prime}=\left|F_{\text {dyn }}(\mathbf{u})\right|^{2}, D(\mathbf{u}),
$$

where $F_{\mathrm{dyn}}(\mathbf{u})$ is a dynamical structure amplitude describing the wave field emerging from the region of one structural unit at the exit face of the crystal. On this basis an analysis has been made of the diffuse scattering effects appearing in the electron diffraction patterns given by magnesium fluorogermanate (Cowley \& Kunzmann, in preparation). For many materials the need for the interpretation of electron diffraction patterns is avoided by the possibility that high-resolution electron microscopy can be used for direct imaging of the arrangement of atoms in thin crystals. This was demonstrated with a resolution of about $3.5 \AA$ by Iijima, S. [J. Appl. Phys. (1971). 42, 5891-5893] and has been applied by him to the study of the crystal structures of a number of mixed oxides showing regular and irregular superlattices and also the study of the form of the defects associated with the non-stoichiometry of these oxides [e.g. Iijima, S. (1973), Acta Cryst. A 29, 18-24; Iijima, S. \& Allpress, J. G. (1974a,b) Acta Cryst. A 30, 22-29, 29-36]. Evidence has been obtained from the direct viewing of metal atom positions concerning the short-range ordering of atoms in oxides and in minerals [Iijima, S., Cowley, J. M. \& Donnay, G. (1973). Tschermaks. Miner. Petrogr. Mitt. 20, 216-224] and also the diffusion processes associated with phase transformations (Iijima \& Allpress, 1974a). There are indications that individual point defects [Iijima, S., Kimura, S. \& Goto, M. (1973). Acta Cryst. A29, 632-636] and defect clusters (Iijima, in preparation) can be imaged clearly and their interactions and ordering can be observed. The present limitations of this technique are those of radiation damage of the specimen by the incident electron beam and the limitation of the interpretable resolution imposed by the values of the spherical-aberration constants of the lenses and the electron wavelength. The use of high-resolution dark-field imaging techniques appears to hold great promise as a means for the study of defects and disorder. If only the diffuse scattering in the diffraction pattern is used to form the image it is only the deviations from the average lattice which will contribute to the image. However, it has been shown [Cowley, J. M. (1973). Acta Cryst. A 29, $529-537,537-560]$ that the image intensity is not necessarily a direct representation of the defect structure and considerable care is needed for the interpretation of such images.

J. Appl. Cryst. (1975). 8, 205

\title{
Ordering and Clustering of Vacancies and Interstitials in Vanadium Monoxide
}

\author{
By B. Andersson, J. Gjønnes, J. Taftø and D. Watanabe* \\ Institute of Physics, University of Oslo, Norway
}

(Received 29 April 1974)

\begin{abstract}
The defect rock-salt type monoxide of vanadium contains large fractions of vacancies on both lattices. Previous studies have demonstrated that interstitial metal atoms also are important in this system and that the defects can be ordered [Andersson, B. \& Gjønnes, J. (1970). Acta Chem. Scand. 24, 2250; Høier, R. \& Andersson, B. (1973). Acta Cryst. A 30, 93; Andersson, B., Gjønnes, J. \& Taftø, J. (1974). Acta Cryst. A30, 216]. Ordering and clustering of defects in the vanadium-oxygen system in the composition range $\mathrm{VO}_{0.55}-\mathrm{VO}_{1.25}$ have been studied further by several electron diffraction methods. These include structure determination of ordered phases, measurement of short-range-order diffuse scattering and determination of structure factors by the critical-voltage and intersecting-Kikuchi-line methods. The results can be summarized thus: At low oxygen content, oxygen vacancies are the most important defects. These cluster in vacancy rows; the ordered phase $\mathrm{V}_{14} \mathrm{O}_{8}$ can be described as an ordered arrangement of such rows. With increasing oxygen content, the content of metal vacancies increases; in the range above $\mathrm{VO}_{1.0}$ there are also metal interstitials. Together with the metal vacancies, these form tetrahedral clusters. Two superstructures, $\mathrm{V}_{52} \mathrm{O}_{64}$ and $\mathrm{V}_{260} \mathrm{O}_{320}$, are described as ordered arrangements of such clusters.
\end{abstract}

\footnotetext{
* On leave from: Department of Physics, Tohuku University, Sendai, Japan.
} 